\title{
Honeycomb-like Neointima of Sirolimus-eluting Stent in Saphenous Vein Graft: Insights from OCT and IVUS
}

Authors: Seiji Koga ${ }^{\text {a }}$, Satoshi Ikeda a ${ }^{\text {, Koji Maemura }}{ }^{\text {a }}$

${ }^{\text {a }}$ Department of Cardiovascular Medicine, Nagasaki University Graduate School of Biomedical Sciences, Nagasaki, Japan

a This author takes responsibility for all aspects of the reliability and freedom from bias of the data presented and their discussed interpretation.

\section{Corresponding author and contact details:}

Satoshi Ikeda

Department of Cardiovascular Medicine, Nagasaki University Graduate School of Biomedical Sciences

1-7-1 Sakamoto, Nagasaki 852-8501, Japan

Tel: +81-95-819-7288; Fax: +81-95-819-7290

Email: sikeda@nagasaki-u.ac.jp

Grant support : none

Conflicts of interest : none

Keywords : Neointima, In-stent restenosis, Optical coherence tomography, Intravascular ultrasound, Drug-eluting stent, Saphenous vein graft 
Drug-eluting stents (DES) have dramatically reduced rates of restenosis and target lesion revascularization compared with bare metal stents (BMS) [1]. However, the first-generation DES, including sirolimus-eluting stents (SES) (Cypher, Cordis, Johnson \& Johnson, Miami, Florida), implanted in native coronary arteries are associated with delayed arterial healing mainly due to hypersensitivity [2]. Here, we present a case with unusual vascular reaction to a SES which was implanted in a saphenous vein graft (SVG), as imaged by in optical coherence tomography (OCT) and intravascular ultrasound (IVUS).

A 39-year-old woman who underwent coronary artery bypass surgery 3 years previously was admitted with symptoms of angina. She was under hemodialysis for 18 years and had coronary risk factors including hypertension and dyslipidemia. Coronary angiography (CAG) showed severe stenosis of a SVG to left anterior descending artery. A SES was implanted to treat this lesion, and subsequent IVUS did not observe stent malapposition. However, after 15 months, her angina occurred again and CAG revealed proximal in-stent restenosis (Fig. A). OCT within the stented segment revealed the diffuse neointimal tissue proliferation with high-intensity layer at surface. In contrast, deep tissue contains heterogeneous low-intensity areas that surrounded and spread behind the stent struts (Fig. B1 - 4). Notably, a honeycomb-like structure separated by high-intensity septa was also identified around the struts (Fig. B4). On the other hand, IVUS demonstrated echolucent or low-echoic areas in the neointima as well as in the tissue behind the stent struts (Fig. C1 - 4). In 
addition, the increases of vessel areas were obvious as compared with previous IVUS findings at time of stent implantation 15 months ago, which implies the occurrence of positive vessel remodeling (Fig. D1 - 4).

It has been reported that the histological features of low-intensity areas in neointimal tissue determined by OCT are excessive inflammation, fibrin accumulation, organized thrombus, extracellular matrix accumulation, or neoatherosclerosis [3]. In contrast, high-intensity structures without backscattering in the surface of neointima visualized by OCT are considered to be fibrin clots [4]. Furthermore, honeycomb-like structures determined by OCT are recanalized organized thrombi [5]. Interestingly, the present IVUS images demonstrated positive vessel remodeling in the stented segment, which is a potential risk for late acquired stent malapposition [6]. Based on these findings, the present OCT images of low-intensity areas may indicate the organized thrombi with partial recanalization and fibrin accumulation that filled spaces around the stent struts, and were covered by fibrin clots in the surface. We suspect that those spaces might be caused by late acquired stent malapposition due to positive vessel remodeling.

\section{Acknowledgement}

The authors of this manuscript have certified that they comply with the Principles of Ethical Publishing in the International Journal of Cardiology [7]. 


\section{References}

1. Moses JW, Leon MB, Popma JJ et al. Sirolimus-eluting stents versus standard stents in patients with stenosis in a native coronary artery. N Engl J Med 2003;349:1315-23.

2. Nakazawa G, Finn AV, Vorpahl M, Ladich ER, Kolodgie FD, Virmani R. Coronary responses and differential mechanisms of late stent thrombosis attributed to first-generation sirolimusand paclitaxel-eluting stents. J Am Coll Cardiol 2011;57:390-8.

3. Landmesser U. Optical coherence tomography (OCT) evaluation after coronary stenting: the "black hole" and other low OCT signal-intensity areas. EuroIntervention 2012;7:1367-71.

4. Fusazaki T, Itoh T, Koeda T, Ogino Y, Matsui H, Nakamura M. Angioscopy and OCT in repeated in-Stent restenosis in saphenous vein graft. J Am Coll Cardiol Img 2010;3:785-6.

5. Kang SJ, Nakano M, Virmani R et al. OCT findings in patients with recanalization of organized thrombi in coronary arteries. J Am Coll Cardiol Img 2012;5:725-32.

6. Hassan AK, Bergheanu SC, Stijnen $\mathrm{T}$ et al. Late stent malapposition risk is higher after drug-eluting stent compared with bare-metal stent implantation and associates with late stent thrombosis. Eur Heart J 2010;31:1172-80.

7. Shewan LG, Coats AJ. Adherence to ethical standards in publishing scientific articles: a statement from the International Journal of Cardiology. Int J Cardiol 2012;161:124-5. 


\section{Figure legends}

\section{Fig. Angiographic, OCT, and IVUS Findings}

Magnified angiographic image of saphenous vein graft (SVG) to left anterior descending artery (A).

In-stent restenosis (a yellow arrow) was observed at proximal portion of a sirolimus-eluting stent (2.5

$\mathrm{mm} \times 23 \mathrm{~mm}$ ) which was implanted 15 months ago (a white dotted line). Optical coherence tomography (OCT) shows a diffuse proliferation of neointima with high-intensity surface (B1 -4). On

the other hand, deep tissue contains heterogeneous low-intensity areas that surrounded and spread behind the stent struts (white arrows) and are clearly demarcated by SVG wall. A honeycomb-like structure separated by high-intensity septa was identified (B4). Intravascular ultrasound (IVUS) shows echolucent (yellow arrowheads) or low-echoic (white arrowheads) areas in neointima and behind struts (C1 - 4). IVUS images of same slices at time of stent implantation 15 months previously confirm absence of stent malapposition (D1 - 4). Vessel areas (VA) measured in C1 - $\mathbf{4}$ are greater than those in D1 - 4, suggesting positive vessel remodeling. Red circles in IVUS images indicate vessel areas. 
Fig.
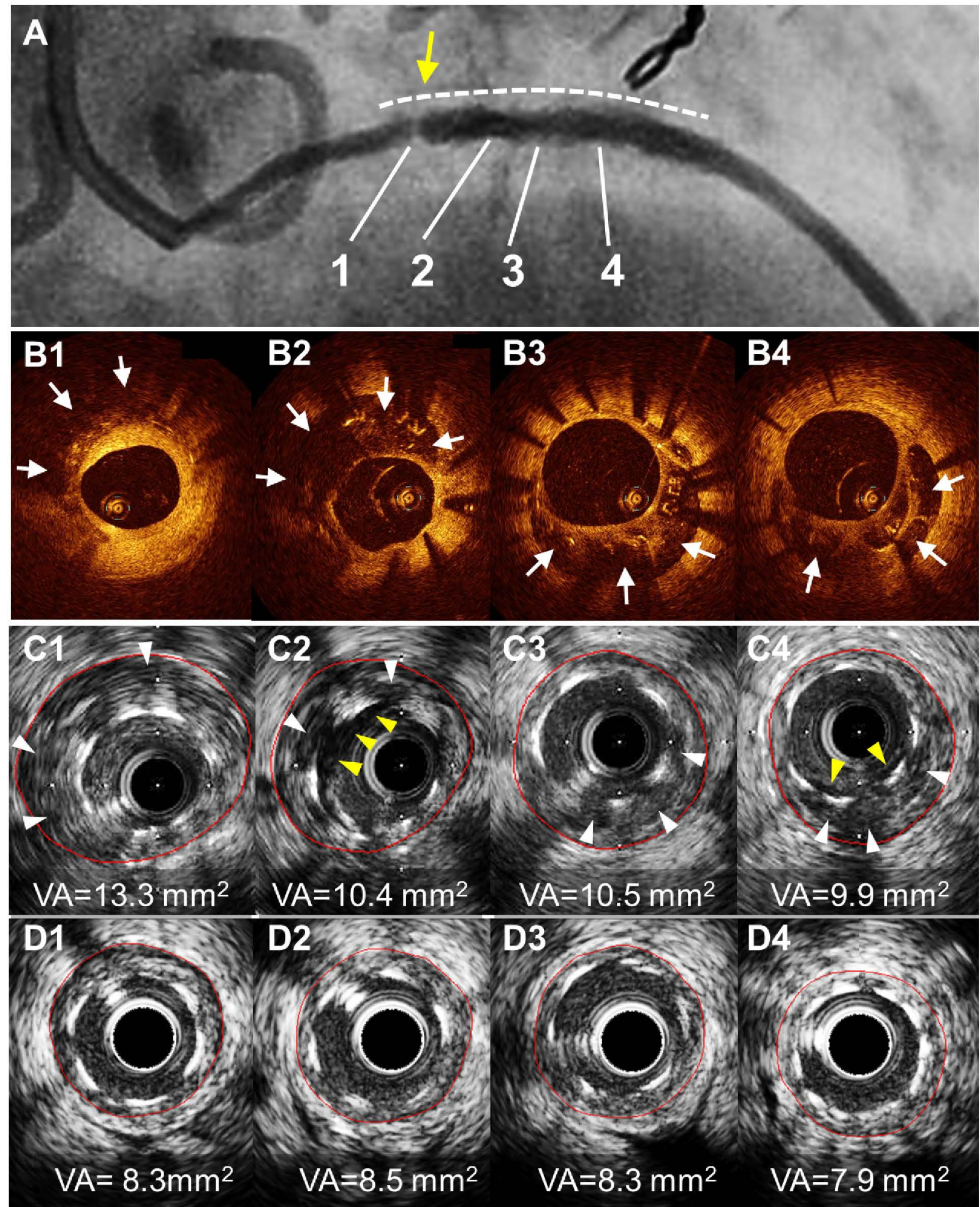